\title{
Realidad de la evaluación de los aprendizajes en la Universidad Católica de EI Salvador
}

| Karla Margarita Fuentes Barillas

Licenciada en Ciencias de la Educación con especialidad en Educación Básica

Docente investigadora, Facultad de Ciencias y Humanidades

Universidad Católica de El Salvador, El Salvador

Email: karla.fuentes@catolica.edu.sv

ORCID: https://orcid.org/0000-0003-3248-6351
Fecha de recepción: $20-01-2021$

Fecha de aceptación: 21-02-2021

\section{Resumen}

La investigación presenta un análisis de la realidad de la evaluación de los aprendizajes en la Universidad Católica de El Salvador. Se abordó desde un enfoque cualitativo, por medio de entrevistas y grupos de enfoque, la forma en que docentes y estudiantes entienden la evaluación y su finalidad. También, se puntualizaron las actividades más frecuentes que se emplean para evaluar los aprendizajes en la Universidad.

Los hallazgos revelan que tanto docentes como estudiantes entienden que la evaluación de los aprendizajes responde a una finalidad educativa. Esto significa que su objetivo es valorar los aprendizajes (teóricos, prácticos y actitudinales) que se adquieren en una asignatura. Además, que las actividades de evaluación cumplen con dicha finalidad, cuando se vinculan directamente a los retos profesionales y sociales de la actualidad.

Palabras clave: Evaluación, aprendizaje, educación superior, finalidad.

\begin{abstract}
he research presents an analysis of the reality of learning assessment at Universidad Católica de El Salvador. A qualitative approach was used, through interviews and focus groups, to analyze the way in which teachers and students understand evaluation and its purpose. Also, the most frequent activities used to evaluate learning at the University were pointed out.

The findings reveal that both teachers and students understand that the assessment of learning responds to an educational purpose. This means that its objective is to assess the theoretical, practical and attitudinal learning acquired in a subject. In addition, that the evaluation activities fulfill this purpose when they are directly linked to the professional and social challenges of today.
\end{abstract}

Key words: Assessment, learning, higher education, educational purpose. 


\section{Introducción}

El concepto de "evaluación", no es exclusivo de la educación. (Flores, 2010). Se evalúa en distintos contextos, con la finalidad de establecer un juicio sobre la idoneidad -o calidad- de algo. (Alcaraz, 2014). Para el caso de la educación, la evaluación puede definirse de distintas maneras según las necesidades u objetivos de una institución (Mora, 2004). El Ministerio de Educación de El Salvador ${ }^{1}$ (MINED, 2008) plantea que: "La evaluación es parte integrante del proceso de enseñanza-aprendizaje. No es el final del proceso sino el medio para mejorarlo, ya que sólo por medio de una adecuada evaluación se podrán tomar decisiones que apoyen efectivamente al alumnado" (p.9). La evaluación de los aprendizajes permite verificar el logro de las finalidades educativas en todos los niveles. El contexto actual marcado por constantes cambios, precisa establecer una reflexión continua sobre qué y cómo se evalúa en la Universidad.

La Universidad Católica de El Salvador ${ }^{2}$ (UNICAES, 2020) cuenta con un reglamento de evaluación del rendimiento académico estudiantil. En el artículo 1 establece que:

La evaluación es un proceso que forma parte del quehacer educativo, planificada y permanente, que permite la recopilación de información a través de procedimientos cualitativos y cuantitativos, con el fin de emitir juicios valorativos que sirvan de base para determinar el nivel de logro alcanzado por el estudiante en los objetivos propuestos, de acuerdo con un referente de crecimiento integral del ser humano que oriente la toma de decisiones académicas. (p. 31)

La definición de "evaluación" de la UNICAES plantea la formación integral de la persona de acuerdo con su ideario educativo. Todas las carreras ofrecidas por la institución incluyen asignaturas orientadas a dicha formación ${ }^{3}$. Dentro del Modelo Educativo de la UNICAES (2020) se concibe la evaluación como "parte de los procesos de aprendizaje ya que su finalidad es identificar, valorar y reforzar el nivel de logros de aprendizaje alcanzado por el estudiante en el proceso de desarrollo personal y profesional" (p. 16). Dentro de este documento, también se reconoce el rol central del docente como mediador de una evaluación integral de los aprendizajes. Además, se reconoce que la evaluación de los aprendizajes es:

un espacio de reflexión y toma de decisiones y no únicamente como un proceso administrativo que hay que cumplir, ya que su quehacer educativo está centrado en el educando. Esto no descarta el hecho de que la evaluación que se hace a los alumnos refleja el nivel y calidad del trabajo educativo que realiza el docente, es decir que de manera

1. Dentro del artículo, la autora hará referencia al Ministerio de Educación, mediante su acrónimo, MINED.

2. La autora hará referencia a la institución mediante su acrónimo, UNICAES.

3. Las asignaturas se dividen en tres tipos: generales, humanísticas y de especialización. 
directa el buen aprendizaje de los alumnos refleja la buena enseñanza del docente y en su defecto, los malos resultados en el aprendizaje denotan la baja calidad de la enseñanza del maestro. (p. 16)

Por lo tanto, se entiende que la evaluación de los aprendizajes cumple con una finalidad administrativa (reportar con datos cuantitativos los resultados de aprendizaje a una entidad pertinente) y una finalidad educativa (orientada al cumplimiento de objetivos educativos).

Sans (2008) reconoce que "La evaluación de los aprendizajes de los alumnos no es simplemente una actividad técnica o neutral, sino que constituye un elemento clave en la calidad del aprendizaje, ya que condicionará la profundidad y el nivel" (p. 8). Lo anterior, alude a un proceso planificado de la evaluación donde se toman en cuenta los objetivos, los contenidos y la metodología sugerida para una asignatura. No obstante, algunos factores como la preparación pedagógica deficiente y la concepción limitada de la evaluación (instrumento de medición para aprobar, reprobar y promover...) podría incidir en cómo se realiza este proceso en la Universidad (Moreno, 2009). A este respecto, a partir del año 2012, desde la Facultad de Ciencias y Humanidades de la UNICAES, se han impartido cursos y diplomados sobre formación pedagógica a docentes de otras facultades. Más recientemente, se imparte un diplomado en "Planificación y Evaluación de los Aprendizajes" y “Competencias Docentes". El propósito es brindar herramientas teóricas y prácticas para que los docentes mejoren su desempeño.

Con base en lo planteado anteriormente, las preguntas orientadoras de la presente investigación fueron: ¿La evaluación de los aprendizajes que se realiza en la Universidad Católica de El Salvador responde a una finalidad educativa o administrativa?, ¿de qué manera identifica cada docente la finalidad de la evaluación de los aprendizajes? y en la práctica, ¿cómo se verifica la finalidad de la evaluación a través de las actividades desarrolladas por los docentes?

La investigación surgió como una primera aproximación sobre el proceso de evaluación de los aprendizajes a nivel universitario en la UNICAES. En cuanto a los beneficiarios del estudio ha sido principalmente la comunidad educativa UNICAES en general y la Facultad de Ciencias y Humanidades en particular; con el objetivo de mejorar los procesos de capacitación docente. Se analizó la correspondencia y claridad entre la finalidad del proceso de evaluación en general y las actividades para verificar los aprendizajes en particular; para incentivar a la toma de decisiones en la que el proceso de evaluación corresponda a una finalidad educativa.

La investigación tuvo como objetivos, analizar la realidad de la evaluación de los aprendizajes en la Universidad Católica de El Salvador. Además, puntualizar los tipos de actividades de evaluación que aplican los docentes y determinar la finalidad que presentan las activi- 
dades de evaluación aplicadas por los docentes en dicha institución.

\section{Metodología}

El proceso de recolección de datos se realizó desde un enfoque cualitativo y descriptivo. $\mathrm{La}$ metodología aplicada durante la investigación permitió profundizar sobre un tema complejo y poco explorado. La muestra fue seleccionada al azar. Se abordó a docentes y estudiantes de las cuatro facultades: Ciencias Empresariales, Ciencias de la Salud, Ciencias y Humanidades e Ingeniería y Arquitectura. Quienes, a su vez, fueron clasificados de acuerdo a tres tipos de asignaturas: generales, humanísticas y de especialización. Además, se determinó llevar a cabo la investigación sobre las prácticas de evaluación de los aprendizajes en asignaturas de segundo y octavo ciclo académico ${ }^{4}$, de carreras presenciales que se imparten en la Universidad Católica de El Salvador, sede Santa Ana. En cuanto a las técnicas e instrumentos utilizados, se detallan a continuación:

Entrevista: Esta técnica fue realizada con 12 docentes de las cuatro facultades ${ }^{5}$ como se muestra en la Tabla 1. El instrumento aplicado fue una guía de entrevista con doce preguntas abiertas, relacionadas a la finalidad y tipos de actividades de evaluación, la relación entre objetivos, contenidos y metodología de las asignaturas; criterios de evaluación según tipo de actividades y proceso de realimentación (ver tabla 1).

Grupos de enfoque: Se realizaron cuatro grupos de enfoque (uno por cada facultad) con seis estudiantes cada uno. En total participaron 24 estudiantes (dos por cada asignatura). Se determinó que los alumnos representaran a los docentes seleccionados para las entrevistas. El instrumento utilizado fue un cuestionario conformado por siete preguntas abiertas. De igual

Tabla 1. Cantidad de docentes entrevistados en cada Facultad (según tipo de asignatura)

\begin{tabular}{|c|c|c|c|c|}
\hline \multirow{2}{*}{ Facultad } & \multicolumn{3}{|c|}{ Tipo de asignatura } & \multirow{2}{*}{$\begin{array}{l}\text { Total por } \\
\text { Facultad }\end{array}$} \\
\hline & General & Humanística & Especialización & \\
\hline $\begin{array}{c}\text { Ciencias } \\
\text { Empresariales }\end{array}$ & 1 & 1 & 1 & 3 \\
\hline $\begin{array}{c}\text { Ciencias } \\
\text { de la Salud }\end{array}$ & 1 & 1 & 1 & 3 \\
\hline $\begin{array}{c}\text { Ciencias y } \\
\text { Humanidades }\end{array}$ & 1 & 1 & 1 & 3 \\
\hline $\begin{array}{l}\text { Ingeniería y } \\
\text { Arquitectura }\end{array}$ & 1 & 1 & 1 & 3 \\
\hline Total general & & & & 12 \\
\hline
\end{tabular}

4. Se determinó tomar en cuenta estos ciclos para conocer la posible variación del proceso de evaluación durante el inicio y el final del estudio de una carrera universitaria.

5. Se aplicó un muestreo no probabilístico por conveniencia debido a las consideraciones expuestas sobre el ciclo académico y tipo de asignatura. 
forma, las preguntas tenían relación con los indicadores del estudio.

\section{Resultados}

\section{Tipos de actividades de evaluación}

Los docentes reconocen la relación de complementariedad entre las actividades de evaluación con los objetivos, contenidos y metodología propuesta para las asignaturas que imparten. En este sentido, los elementos de la planificación didáctica giran en torno al tipo de materia que el docente desarrolla (general, humanística, de especialización); la metodología didáctica se adapta en función de diversos factores, por ejemplo: la cantidad de estudiantes inscritos, sus aprendizajes previos, el tipo de profesión en la que se estén formando y el nivel de avance en la carrera. Partiendo de lo anterior, los docentes acoplan y planifican las actividades de evaluación. También, afirman que utilizan las herramientas tecnológicas como apoyo para el desarrollo de las clases y evaluaciones.

Las actividades de evaluación son variadas. Los docentes expresaron que las principales actividades para evaluar los aprendizajes de los estudiantes son los exámenes teóricos (escritos y virtuales) y las exposiciones sobre contenidos específicos y sobre proyectos asignados. También, se incluyen la resolución de guías de ejercicios, estudios de caso, realización de videos, talleres, diseño de diagramas, investigaciones bibliográficas, visitas técnicas y cine foro. Además, las entregas de estas actividades son individuales y grupales. En cuanto a los estudiantes, reconocieron que la variedad de evaluaciones que se realizan generan mejores aprendizajes y les ayuda a desarrollar sus habilidades.

Tanto docentes como estudiantes, coincidieron en que las actividades de evaluación que generan mayores aprendizajes son las que están vinculadas al ejercicio profesional. La mayoría de esas actividades tienen que ver con la capacidad de análisis y la aplicación de los conocimientos a situaciones concretas -y reales- presentes en el campo laboral. Además, la utilización de la tecnología y los recursos materiales específicos de cada profesión (uso de laboratorios para química e informática), propician un mejor desempeño de los estudiantes. Los alumnos valoran que sus actividades de evaluación estén enfocadas a desarrollar en ellos no solo el dominio teórico y práctico de sus especialidades, sino también la adquisición de competencias blandas, o actitudes que les permitan enfrentarse a diversas situaciones en su desempeño profesional.

Con respecto a los criterios u orientaciones para evaluar las actividades, estos se pueden clasificar en generales y específicos. Los docentes establecen una serie de orientaciones que guían los aprendizajes y/o productos esperados por parte de los estudiantes. Los criterios generales aplican para todo tipo de evaluación, mientras que los criterios específicos, derivan de la naturaleza propia de los tipos de actividades (ver tabla 2).

Cada docente establece con antelación, los requerimientos para las actividades en cuanto in- 
Tabla 2. Clasificación de criterios para las actividades de evaluación en la Universidad Católica de El Salvador

\begin{tabular}{|c|c|c|c|}
\hline \multirow{2}{*}{ Criterios generales } & \multicolumn{3}{|c|}{ Criterios específicos } \\
\hline & Actividades escritas & Actividades orales & $\begin{array}{c}\text { Actividades } \\
\text { prácticas }\end{array}$ \\
\hline $\begin{array}{l}\text {-Dominio de } \\
\text { vocabulario técnico } \\
\text {-Creatividad } \\
\text {-Nivel de dificultad } \\
\text { de la actividad } \\
\text {-Responsabilidad } \\
\text {-Puntualidad en la } \\
\text { entrega } \\
\text {-Calidad del } \\
\text { contenido } \\
\text {-Orden }\end{array}$ & $\begin{array}{l}\text {-Aplicación de } \\
\text { conceptos a } \\
\text { situaciones concretas } \\
\text {-Nivel de análisis } \\
\text {-Argumentación } \\
\text {-Originalidad } \\
\text {-Redacción } \\
\text {-Ortografía } \\
\text {-Coherencia en la } \\
\text { estructura de un } \\
\text { texto de } \\
\text {-Utilización } \\
\text { fuentes bibliográficas }\end{array}$ & $\begin{array}{l}\text {-Utilización de } \\
\text { vocabulario técnico } \\
\text {-Dominio del tema } \\
\text {-Presentación } \\
\text {-Seguridad en la } \\
\text { expresión } \\
\text {-Paralingüística } \\
\text {-Claridad } \\
\text {-Argumentación }\end{array}$ & $\begin{array}{l}\text {-La funcionalidad de } \\
\text { un proyecto } \\
\text {-Manejo } \\
\text { situaciones } \\
\text { imprevistas } \\
\text {-Utilización de } \\
\text { recursos tecnológicos } \\
\text {-Conducta } \\
\text {-Trabajo en equipo }\end{array}$ \\
\hline
\end{tabular}

dicaciones, criterios y condiciones para las entregas. En la mayoría de los casos, se presentan rúbricas de evaluación que especifican el nivel de aprendizaje que se espera de los estudiantes. Además, en algunas ocasiones los docentes toman acuerdos en conjunto con los discentes sobre las actividades de evaluación.

Procurar la objetividad y la justicia en la evaluación es la principal recomendación de los estudiantes para los docentes. En primer lugar, los estudiantes sugieren que se asignen ponderaciones justas. Por un lado, de acuerdo al nivel de complejidad de una actividad y, por otro lado, conforme a la calidad de lo que se en- trega. En segundo lugar, recomiendan que los docentes mantengan la ponderación, las actividades y los criterios de evaluación informados al inicio, sin cambiarlos mientras se califica o en el transcurso de un período. Para el caso de las actividades grupales, deberían considerar el desempeño individual de los integrantes, para evitar injusticias en las calificaciones.

\section{Finalidad de las actividades de evaluación}

Sobre la comprensión del concepto de "evaluación de los aprendizajes", los docentes lo entienden como un proceso. Una de las funciones de la evaluación, es que permite determinar el nivel de logro de aprendizajes tanto teóricos, 
prácticos e incluso actitudinales, según el tipo de asignatura. También, la evaluación es una práctica que se realiza de manera permanente, no solo al final de un período. Está orientada a verificar y determinar la consolidación de los aprendizajes en los estudiantes durante el curso de una asignatura; por lo tanto, la principal finalidad de la evaluación es el aprendizaje de los estudiantes en cuanto a conocimientos y habilidades específicas según asignaturas y profesiones. Los estudiantes coinciden en que la finalidad de la evaluación es verificar el grado en que ellos han adquirido los conocimientos en una asignatura, de cara a su desarrollo profesional. No obstante, reconocen que la calificación numérica no define en su totalidad lo que han aprendido.

En cuanto a los resultados de aprendizaje en cada período, estos permiten orientar el trabajo docente. Posterior al proceso de evaluación, los catedráticos analizan los resultados obtenidos por los estudiantes: en primer lugar, para verificar el cumplimiento de los objetivos de la asignatura y, en segundo lugar, para decidir sobre la continuidad en las metodologías utilizadas o los tipos de actividades de evaluación realizadas. A partir de esto, determinan si es necesario hacer ajustes en su labor, a fin de guiar a los estudiantes hacia la consolidación de los aprendizajes por medio de acciones concretas. Ambos sujetos de estudio reconocieron que el caso más común es la entrega y resolución de un examen teórico. Además, las decisiones tomadas a partir de los resultados de aprendizaje implican acuer- dos entre docentes y estudiantes, estructuradas en un conjunto de pasos. El primero, es la toma de contacto grupal, analizando el desempeño en las actividades evaluadas durante un período, para detectar fortalezas y debilidades; el segundo, es establecer mecanismos de refuerzo académico, según necesidades; y el tercero implica revisar la planificación establecida al inicio del ciclo para determinar cambios o ajustes en metodología y actividades.

Los estudiantes estiman que sus docentes les incentiven a continuar aprendiendo, al reconocer su buen desempeño en las evaluaciones. Esta realidad se evidencia a través de la realimentación, que se realiza con cada devolución de actividades. En algunos casos, las rúbricas de evaluación incluyen observaciones por parte del docente, para informar sobre el nivel de cumplimiento de los criterios establecidos. También, algunas actividades implican una secuencia de entregas ponderadas en cada período. En ese caso el resultado final también es evaluado al término del ciclo académico.

\section{Discusión}

Con la presente investigación, se logró responder a las interrogantes: ¿La evaluación de los aprendizajes que se realiza en la Universidad Católica de El Salvador responde a una finalidad educativa o administrativa?, ¿cómo el docente identifica la finalidad de la evaluación de los aprendizajes?, y ¿De qué manera se verifica la finalidad de la evaluación a través de las actividades desarrolladas por los docentes?, 
analizando las perspectivas de los catedráticos y de los estudiantes.

En efecto, la evaluación de los aprendizajes en la UNICAES responde a una finalidad educativa. Esto significa que la intención detrás del proceso evaluativo es determinar el logro de los aprendizajes esperados en una asignatura. La calificación numérica es la evidencia inmediata de un resultado de aprendizaje; sin embargo, es más importante que los estudiantes adquieran los conocimientos y habilidades específicos para su profesión. A este respecto, Fernández (2014), reconoce que nuestra cultura confunde evaluación y calificación, y que administrativamente, los resultados de aprendizaje también son utilizados para juzgar el desempeño de los docentes, con base a alumnos aprobados y reprobados.

Los programas de estudio plantean una serie de objetivos, contenidos y propuestas metodológicas según el tipo de asignatura; no obstante, el docente decide según considera conveniente, cómo llevar a cabo la evaluación de los aprendizajes. Los elementos que inciden en la estrategia evaluativa tienen que ver con las diferencias individuales de los estudiantes (habilidades, tipos de aprendizaje, hábitos de estudio, etc.) así como los aspectos administrativos en la institución (duración de períodos y la cantidad de alumnos inscritos en una asignatura). La UNICAES cuenta con un reglamento de evaluación del rendimiento académico estudiantil, en el que se establecen los aspectos administrativos y académicos para el desarrollo de esta práctica. Los docentes que participaron en la investigación manifestaron conocer dichos lineamientos.

El tipo de actividades que posibilitan la concreción de la finalidad educativa de la evaluación, son las que tienen una vinculación directa con la realidad profesional. Brown y Glasner (2007) afirman que los exámenes escritos aún predominan como estrategia evaluativa en la Universidad, lo cual también fue un hallazgo en el presente estudio. En este sentido, las autoras plantean que se debe optar por la realización de diversas actividades, en las que se tomen en cuenta experiencias significativas de aprendizaje de los estudiantes y se establezca una interacción junto a los docentes.

Los constantes cambios en la época actual demandan que la Universidad adopte un rol activo para el desarrollo social, es necesario repensar las formas en que se enseña, se aprende y se evalúa. Tobón, Rial, Carretero y García (2006) afirman que el currículo universitario integra diferentes tipos de saberes en los que se deberían tomar en cuenta actividades y problemas profesionales y sociales. En esta misma línea, el enfoque curricular por competencias plantea que el proceso educativo esté centrado en el desarrollo de habilidades y la aplicación de los conocimientos a situaciones concretas.

En función de los resultados de aprendizaje la evaluación también puede ser motivadora. La realimentación es el medio más pertinente para la mejora continua. Los estudiantes necesitan 
saber si su desempeño es correcto o necesitan hacer algo más para lograr los aprendizajes esperados. Sin embargo, la evaluación implica principios como la ética, la objetividad, el respeto y la justicia. Todo ello mediado por un proceso de comunicación y apertura para que la realimentación cumpla con su cometido de orientar la mejora y el aprendizaje.

A partir de los hallazgos revelados en el proceso de evaluación de los aprendizajes en la UNICAES, se pueden brindar las siguientes recomendaciones:

\section{a. La evaluación de los aprendizajes podría} planificarse en función de las demandas sociales en la actualidad. La actualización docente es una herramienta que le permite estar informado de nuevas tendencias en la disciplina que imparte. Además, la planificación docente en conjunto podría dotar de herramientas para impulsar una visión global de la realidad profesional. Por ejemplo, cuando los catedráticos que impartirán asignaturas a un mismo grupo de estudiantes consideren la posibilidad de fomentar la transversalidad de las disciplinas y relacionarlas en las actividades de evaluación. Sería pertinente que se establezcan comunidades de aprendizaje de docentes para planificar actividades de evaluación. Otro aspecto a considerar es la mediación en el uso de la tecnología en el proceso de evaluación. Mendoza y Nájera (2017) consideran que la evaluación del aprendizaje en entornos virtuales, precisa de una adecuada planificación y conocimiento de las herramientas y recursos tecnológicos. Para el caso de la UNICAES, la plataforma Moodle cuenta con diversidad de actividades y recursos que sirven de apoyo en la enseñanza.

b. Adoptar nuevas formas de enseñar, aprender y evaluar. La tradicional forma de concebir el proceso educativo ha estado centrada en el dominio conceptual de los saberes. Una propuesta innovadora de la educación contempla la aplicación de nuevos enfoques metodológicos y la integralidad de la persona. Sin menoscabo de la importancia de los saberes conceptuales, mientras el currículo esté basado en contenidos, podría limitar el desarrollo de habilidades. Una recomendación es que las unidades encargadas de ofrecer capacitación docente implementaran una propuesta de formación basada en competencias; la cual, en aportes de Tobón, Pimienta y García (2010), toma en cuenta el bagaje de conocimientos del estudiante y lo guía hacia un proceso de metacognición permanente.

\section{c. Involucrar a los estudiantes en el proce-} so de la planificación de la evaluación de los aprendizajes. Si bien es cierto, la evaluación en muchos casos constituye un elemento de poder e imposición, los docentes pueden tomar en cuenta las sugerencias y valoraciones de sus estudiantes. Independientemente de la modalidad de una evaluación, los mecanismos como la autoevaluación y la coevaluación también pueden ser útiles. En palabras de Fernández (2014) son elementos de la evaluación inclusiva que toma en cuen- 
ta las diferencias individuales de los estudiantes y propicia espacios para la reflexión y valoración del proceso de aprendizaje. No obstante, el docente tiene un rol activo en la formación de actitudes como la objetividad, la justicia y la ética de la evaluación. La realimentación mediada por un ambiente de confianza y respeto, ayuda al estudiante a incorporar nuevos aprendizajes o atender sugerencias. Es necesario realimentar procesos, más que resultados; descripciones de logros y sus formas de alcanzarlos, más que calificaciones numéricas.

Finalmente, a partir de la realización del presente estudio, surgen nuevas interrogantes: ¿De qué manera la evaluación de los aprendizajes propicia la formación de habilidades en los estudiantes?, ¿cómo evaluar aprendizajes en función de los retos actuales y futuros? ¿Qué implica una evaluación del aprendizaje inclusiva en la Universidad? Las cuales se podrán abordar en futuras investigaciones.

\section{Referencias}

Alcaraz, N. (2015). Aproximación Histórica a la Evaluación Educativa: De la Generación de la Medición a la Generación Ecléctica. Revista Iberoamericana de Evaluación Educativa, 8 (1), 11-25. https://dialnet.unirioja.es/descarga/articulo/5134142.pdf

Álvarez, I. (2008). Evaluación del aprendizaje en la universidad: una mirada retrospectiva y prospectiva desde la divulgación científica. Electronic Journal of Research in Educational Psychology, 6 (1), 235-271. https://www.redalyc.org/pdf/2931/293121940013.pdf

Arribas, J. (2017). La evaluación de los aprendizajes. Problemas y soluciones. Profesorado. Revista de Currículum y Formación de Profesorado, 21 (4), 381-404. https://www.redalyc.org/ pdf/567/56754639020.pdf

Blanco, O. (2004). Tendencias en la Evaluación de los Aprendizajes. Revista de Teoría y Didáctica de las Ciencias Sociales, (9), 111-130. https://www.redalyc.org/pdf/652/65200907.pdf

Bowden, J. y Marton, F. (2014). La Universidad un espacio para el aprendizaje. Más allá de la Calidad y la Competencia. Narcea Ediciones.

Brown, S. y Glasner, A. (2007). Evaluar en la Universidad. Problemas y nuevos enfoques. Narcea Ediciones.

Cabrerizo, J. y Castillo, S. (2010). Evaluación educativa de aprendizajes y competencias. Pearson Educación, S.A.

Fernández, A. (2014). Evaluando la evaluación de los aprendizajes. UFG Editores. 
Flores, C. (2010). Evaluación de los aprendizajes en la universidad. En blanco y negro. Revista sobre docencia Universitaria, 1 (1), 1-6. http://revistas.pucp.edu.pe/index.php/enblancoynegro/article/view/2189

Gómez, M. y Quesada, V. (2017). Coevaluación o evaluación compartida en el contexto universitario: La percepción del alumnado de primer curso. Revista Iberoamericana de Evaluación Educativa, 10 (2), 9-30. https://doi.org/10.15366/riee2017.10.2.001

Guzmán, J. (2010). La evaluación de los aprendizajes vista desde los profesores efectivos que enseñan psicología. Sinéctica, (34), 1-15. http://www.scielo.org.mx/scielo.php?script=sci_arttext\&pid=S1665-109X2010000100010\&lng=es\&tlng=es.

Hermann, J. y Joakim, M. (2019). Informe de mejores prácticas institucionales Proyecto de USAID de Educación Superior para el Crecimiento Económico.

Mendoza, G. y Nájera, L. (2017). La evaluación del aprendizaje en entornos virtuales en la Universidad Católica de El Salvador. Anuario de Investigación, 6, 151-160. http://www.diyps. catolica.edu.sv/wp-content/uploads/2017/09/11EvaluacionAN2017.pdf

Ministerio de Educación El Salvador. (2008). Evaluación al servicio del aprendizaje: Evaluación por competencias. Ministerio de Educación.

Ministerio de Educación República de El Salvador. (2015). Evaluación al Servicio del Aprendizaje y del Desarrollo. Ministerio de Educación.

Mora, A. (2004). La evaluación educativa: Concepto, períodos y modelos. Revista Electrónica Actualidades Investigativas en Educación, 4 (2),0. https://www.redalyc.org/pdf/447/44740211.pdf

Moreno, T. (2009). La evaluación del aprendizaje en la universidad. Tensiones, contradicciones y desafíos. Revista Mexicana de Investigación Educativa, 14(41), 563-591. http://www.scielo.org.mx/pdf/rmie/v14n41/v14n41a10.pdf

Moreno, T. (2016). Evaluación del aprendizaje y para el aprendizaje Reinventar la evaluación en el aula. http://www.casadelibrosabiertos.uam.mx/contenido/contenido/Libroelectronico/ Evaluacion_del_aprendizaje_pdf

Pimienta, J. (2008). Evaluación de los aprendizajes un enfoque basado en competencias. Pearson Eduaction. http://biblioteca.udgvirtual.udg.mx:8080/jspui/bitstream/123456789/2645/1/ Evaluaci\%C3\%B3n\%20de\%20los\%20aprendizajes.\%20Un\%20enfoque\%20basado\%20 en\%20competencias.pdf 
Sánchez, J. (2011). Evaluación de los aprendizajes universitarios: una comparación sobre sus posibilidades y limitaciones en el Espacio Europeo de Educación Superior. Revista de Formación e Innovación Educativa Universitaria, 4 (1), 40-54. https://dialnet.unirioja.es/servlet/ articulo? codigo $=3655293 \&$ orden $=296706 \&$ info $=$ link

Sans, A. (2008). La evaluación de los aprendizajes: Construcción de instrumentos. Ediciones Octaedro. Santos, M. (2016). La Evaluación como Aprendizaje. Cuando la flecha impacta en la diana. Narcea.

Tobón, S., Pimienta, J. y García, J. (2010). Secuencias didácticas: aprendizaje y evaluación de competencias. Pearson Educación.

Tobón, S., Rial, A., Carretero, M. y García, J. (2006). Competencias, calidad y educación superior. Cooperativa Editorial Magisterio.

Universidad Católica de El Salvador. (2019). Modelo Educativo de la Universidad Católica de El Salvador.

Universidad Católica de El Salvador. (2020). Anuario Académico 2020. https://drive.google.com/ file/d/1sfNHN8MpAxlyAyOu4ePMHBPsQdB1s_-z/view

Universidad Central de Chile, (2017). Manual de apoyo docente. Evaluación para el aprendizaje. https://www.postgradosucentral.cl/profesores/download/manual_evaluacion.pdf

Universidad de Valencia. (s.f.). La evaluación de los estudiantes en la Educación Superior Apuntes de buenas prácticas. https://www3.uji.es/ betoret/Formacion/Evaluacion/Documentacion/La\%20evaluacion\%20estudiantes\%20en\%201a\%20ESuperior\%20UV.pdf 